\title{
A Pre-transplant Blood-based Lipid Signature for Prediction of Antibody-mediated Rejection in Kidney Transplant Patients
}

\author{
Monther Al sultan \\ Virginia Commonwealth University \\ Daniel Contaifer Jr. \\ Joshua Morriss
}

See next page for additional authors

Follow this and additional works at: https://scholarscompass.vcu.edu/gradposters

Part of the Pharmacy and Pharmaceutical Sciences Commons

\section{Downloaded from}

Al sultan, Monther; Contaifer, Daniel Jr.; Morriss, Joshua; Alshammari, Suad; Stern, Jeffrey; Bobba, Sindhura; Kimball, Pamela; King, Anne; Kumar, Dhiren; Levy, Marlon; Gupta, Gaurav; and Wijesinghe, Dayanjan, "A Pre-transplant Blood-based Lipid Signature for Prediction of Antibody-mediated Rejection in Kidney Transplant Patients" (2020). Graduate Research Posters. Poster 54.

https://scholarscompass.vcu.edu/gradposters/54

This Poster is brought to you for free and open access by the Graduate School at VCU Scholars Compass. It has been accepted for inclusion in Graduate Research Posters by an authorized administrator of VCU Scholars Compass. For more information, please contact libcompass@vcu.edu. 
Authors

Monther Al sultan, Daniel Contaifer Jr., Joshua Morriss, Suad Alshammari, Jeffrey Stern, Sindhura Bobba, Pamela Kimball, Anne King, Dhiren Kumar, Marlon Levy, Gaurav Gupta, and Dayanjan Wijesinghe 
The complex biochemistry of human biological systems has been operationally broken down into a set of large molecular categories'. The metabolome, as it is termed, includes four are an integral structural component of cell membranes, play a significant role in energy storage, are involved in a variety of signaling pathways and intersect with other classes of compounds in the metabolome ${ }^{1}$. The lipidome has the ability to influence membrane mediated events's. Distinct lipid profiles have been identified in normal and pathologic conditions, and in response to specific therapeutic interventions. One such intervention is renal transplantation, the treatment of choice for
End Stage Renal Disease (ESRD) shortage of suitable organ donors, and resultant organs, creates a marked supply and demand discrepancy leaving many patients on the waiting list for prolonged periods of time ${ }^{4}$ Current immunosuppression protocols result in a substantial decrease in T-cell mediated rejection at the cost of long term mmunosuppression, with its resultant adverse effects including opportunistic infections ${ }^{4}$, graft damage, and metabolic significant effect on suppressing antibody mod not have a significant effect on suppressing antibody mediated rejection
(AMR) a major cause of graft loss 6 . Management of immunosuppression for individual patients is currently generalized based on protocols. Presently available biomarkers like donor-specific antibodies and degree of sensitization have proven to be inadequate to predict rejection ${ }^{6}$. Thus, there is an unmet need for biomarkers which could allow for better risk stratification to enhance the benefit and limit the risk of the
immunosuppression therapy for individual patients.

The study population consisted of 16 consecutive patients Who developed antibody-mediated rejection within 2 years of idney transplant and 29 stable control (SC) patients who did not develop rejection at any point of post- transplant follow-up. Serial plasma samples are collected and stored at Time 1 (T1 - preall patient's post-transplant as part of an IRB approved biobank protocol at pur institution Indication biopsies were performed for acute allograft dysfunction defined as a rise in creatinine $>20 \%$ above baseline, serum creatinine nadir $\geq 2.0 \mathrm{mg} / \mathrm{dL}$ posttransplant; or delayed graft function $>21$ days post-transplant. Surveillance biopsies were performed in patients with a positive low-cytometric crossmatch ( $\mathrm{T}$ or B >100 mean channel shifts) and/or presence of pre- formed donor-specific antibody [DSA;

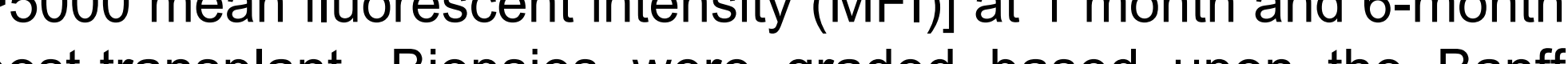
post-transplant. Biopsies were graded based upon the Banff plasmapheresis with intravenous immunoglobulin (IVIG). Preransplant complement-dependent cytotoxicity (CDC) assays and three-color flow- cytometric cross matching (FCXM) were performed for all patients at the time of transplant. Donorspecific antibodies (DSA) were analyzed using the Luminex platform.

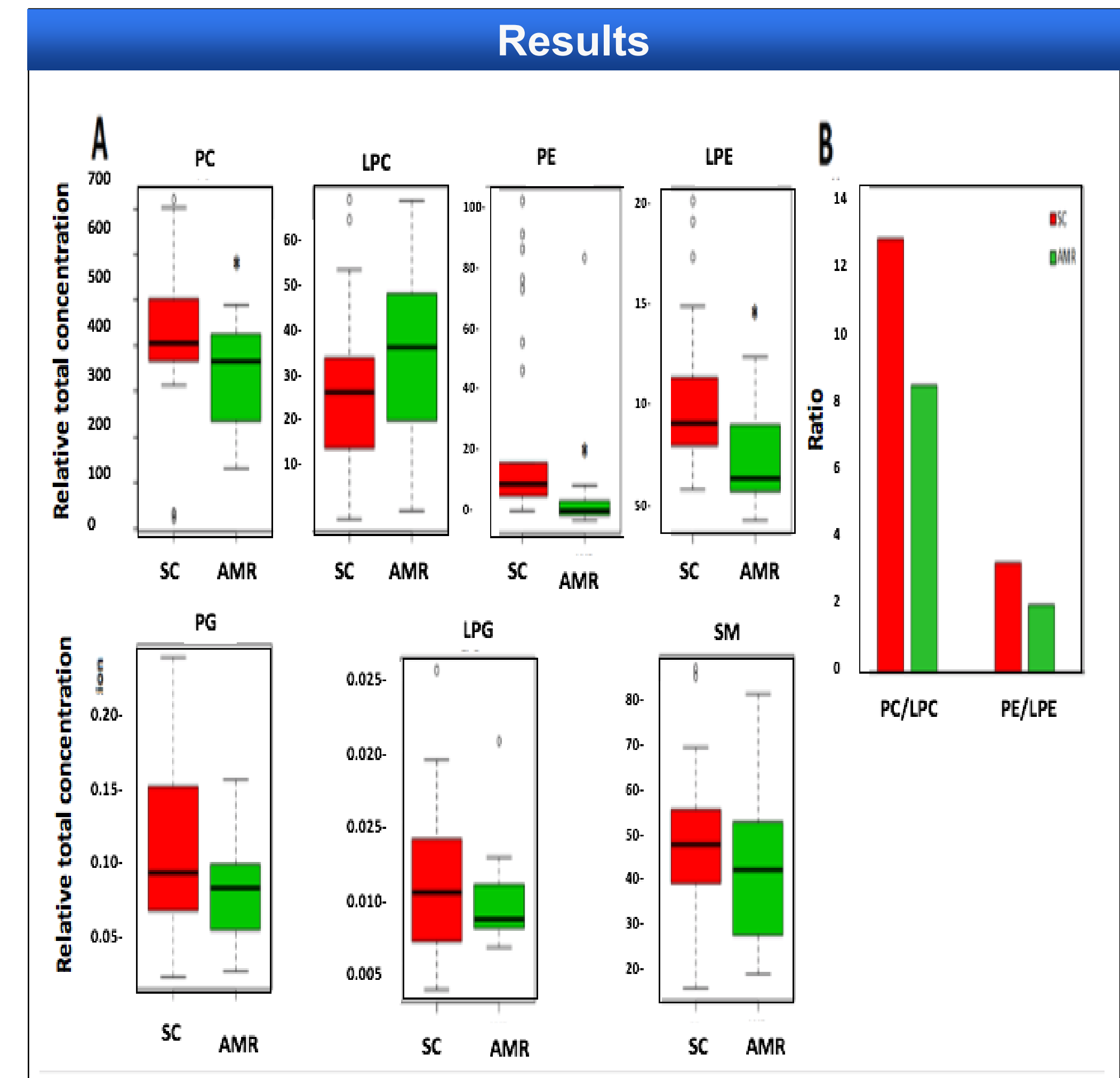

Figure 1: Significant differences are observed among
phospholipids at T1 between SC and AMR. A) AMR group showed a phospholipids at T1 between SC and AMR. A) AMR group showed a
significant lower concentration of PC, PE, and LPE. There was a trend significant lower concentration of $P C, P E$, and $L P E$. There was a trend
towards higher levels of $L P C$ in AMR. B) The ratio of PLs degradation to produce LPLS is an indication of PLA 2 activity with lower values suggesting higher activity. AMR group presented lower ratio for both more PC/LPC and PE/LPE. Suspected outliers are indicated by open
circles in the box plot Green rectangles represent AMR and the Red circles in the box plot. Green rectangles represent AMR and the Red
rectangles represent SC. ${ }^{*}$ indicates significant differences with $p<0.05$.

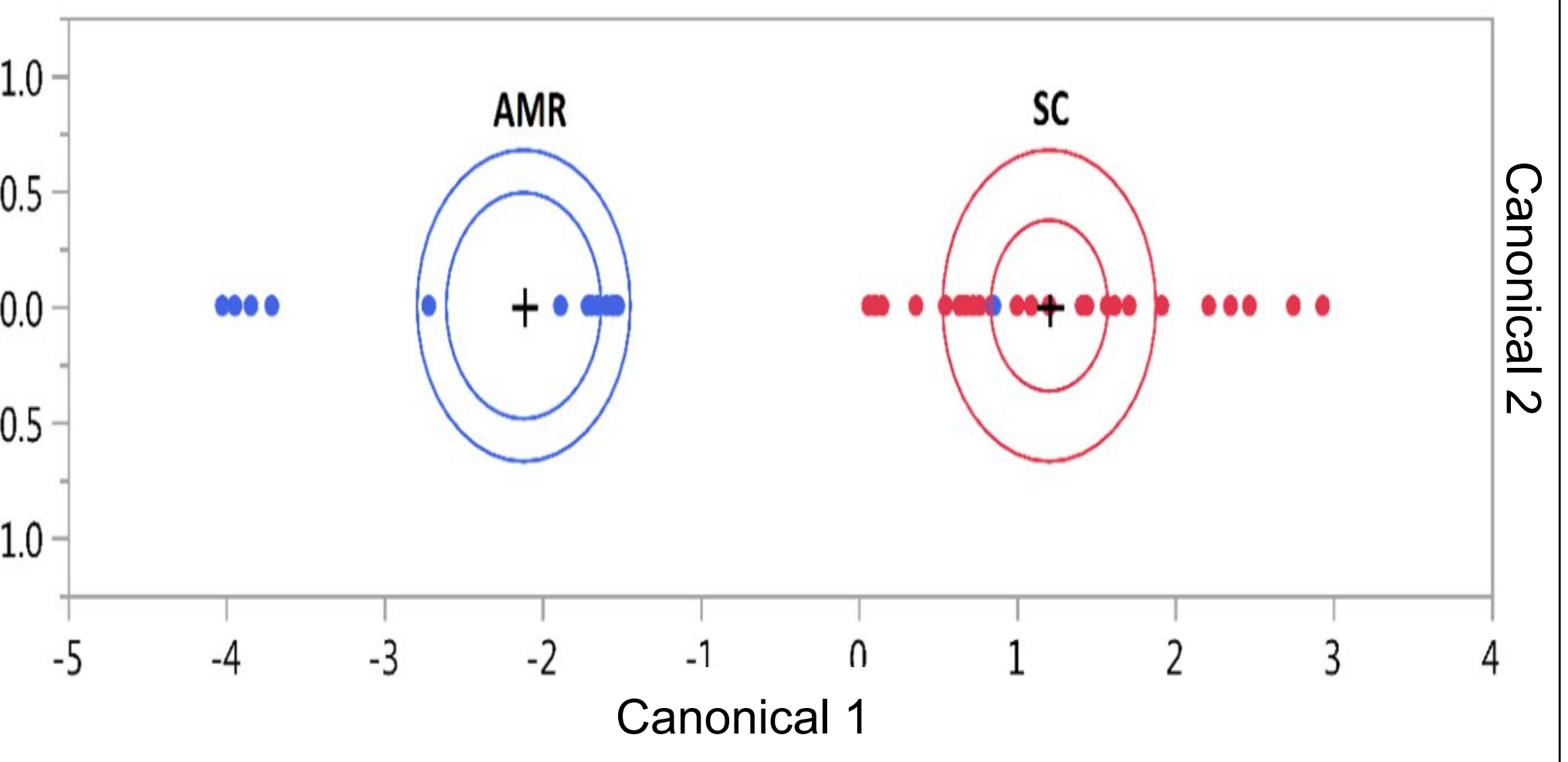

Figure 2: The RLDA model generated using 4 lipids and DSA demonstrate good separation between AMR and SC groups. The RLDA plot shows the clear separation of the patients in the two groups
based on the Mahalanobis distance. This method determines whether based on the Mahalanobis distance. This method determines whether
the selected predictors can separate the distinct categories and reveals the presence of outliers in in the AMR and SC groups. Blue dot among he red dots indicates the one misclassified patient based in the
predictive model. Internal ellipse indicates the $95 \%$ confidence region to contain the true mean of the group. External ellipse indicates the region estimated to contain $50 \%$ of group' population.

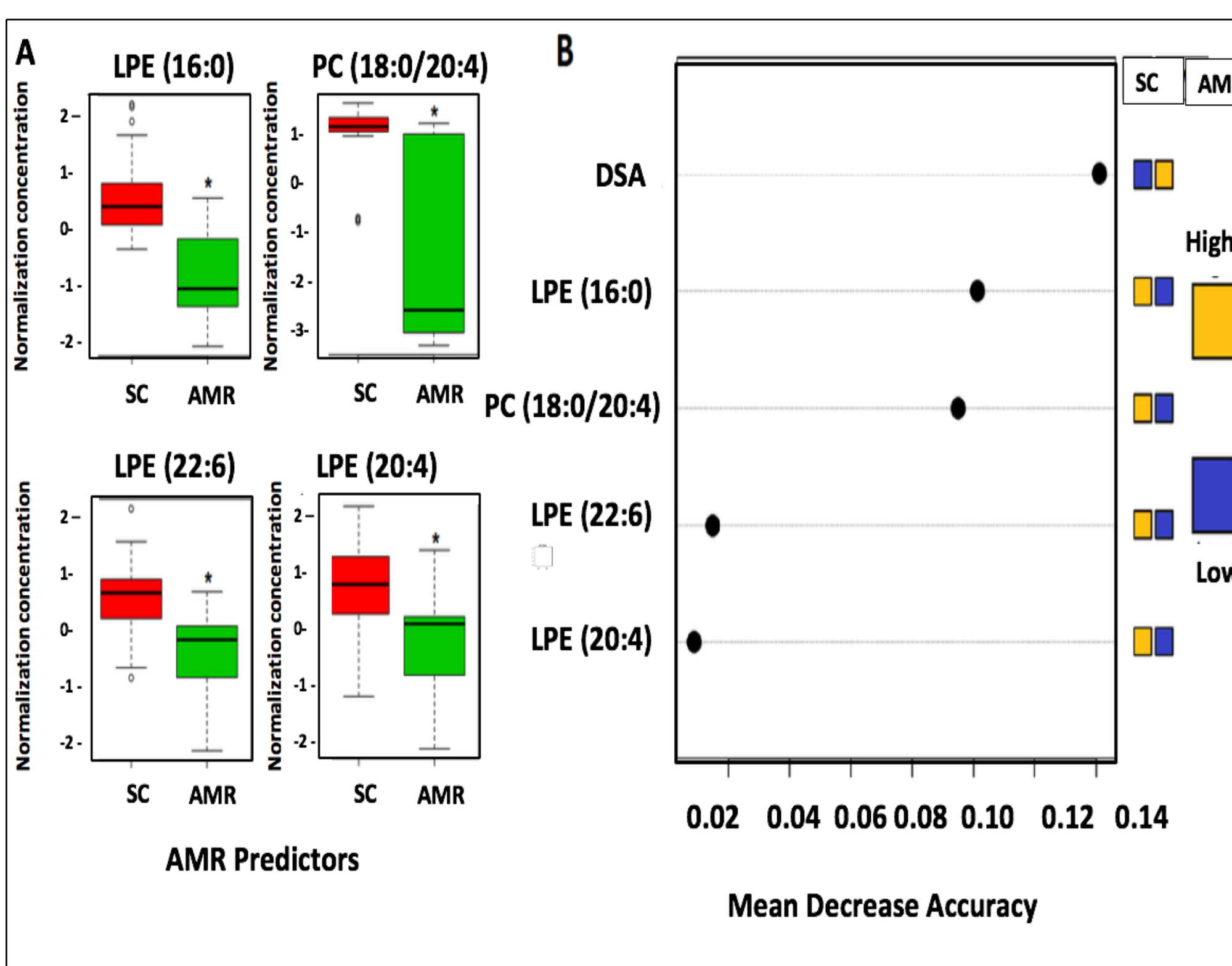

Figure 4: Lipids predict potential for AMR on the day of transplant. A) Box plot of normalized concentration shows that AMR group has lower concentration of the lipids predictors.
Suspected outliers are represented as open circles that appear outside the whiskers. The validation method showed that the transplant with $0.022 \mathrm{OOB}$ error. The mean Decrease Accuracy method shows that DSA is the more important predictor, followed by LPE (16:0) and PC (18.0/20.4) and hey independently could be

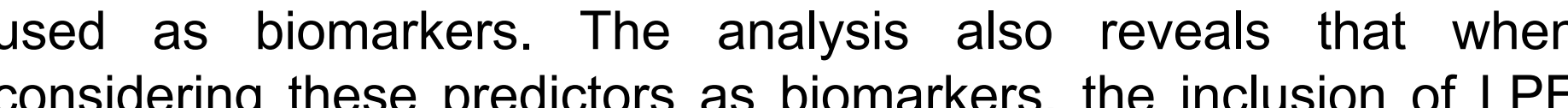
(20:4) and LPE (22:6) does not add any predictive power, and rather must be use to compose the RLDA model. ${ }^{*}$ indicates
significant differences with $p<0.01$.

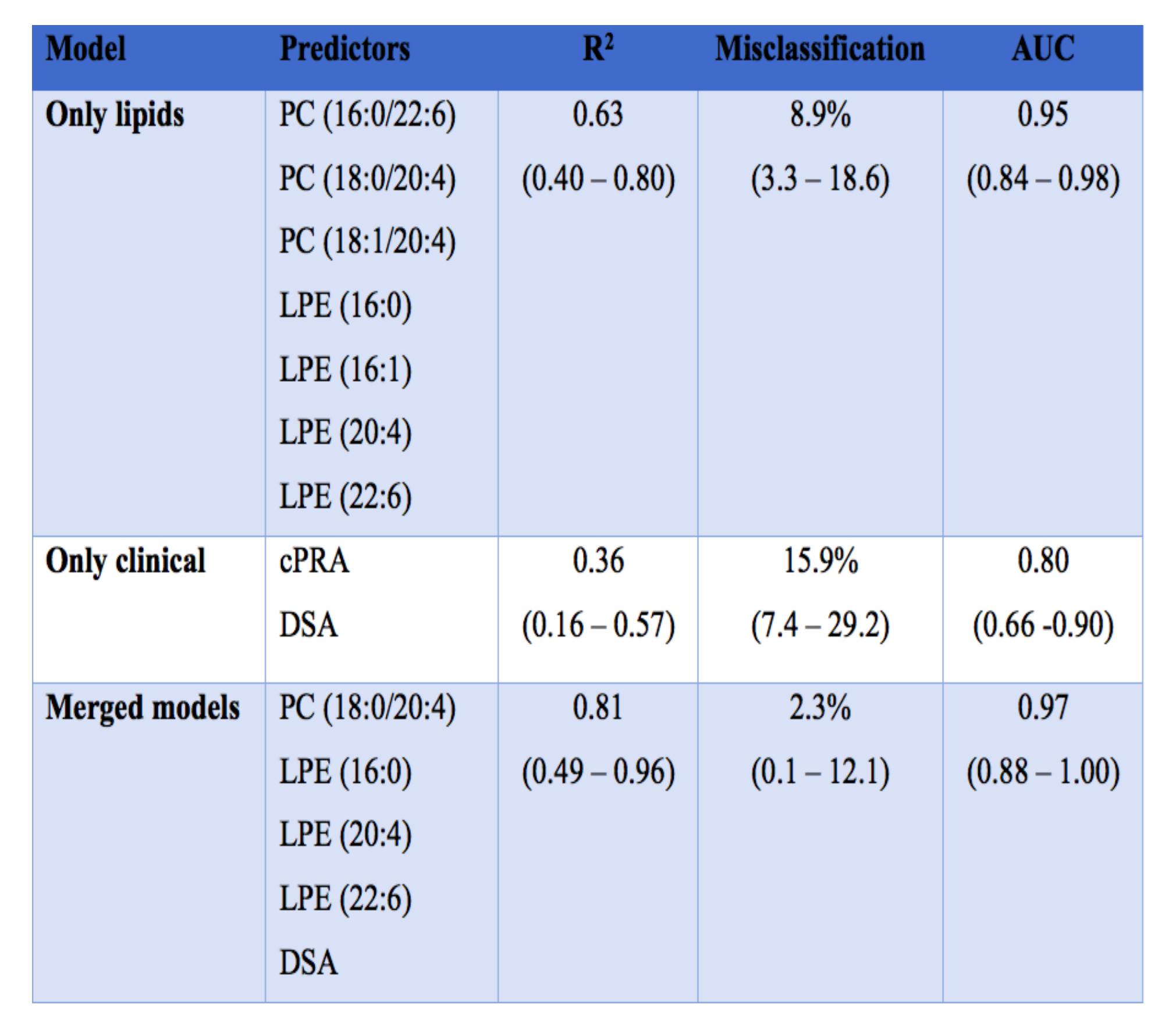

Table 1 - Predictors of Rejection at the Time of Transplant. Bootstrap validation with $95 \%$ Confidence intervals is included for RLDA estimates and area under the curve (AUC). CPRA: Calculated
Panel Reactive Antibody; DSA: donor specific antibodies; GFR: Estimated glomerular filtration rate (mL/min/1.73m2); SC: Stable

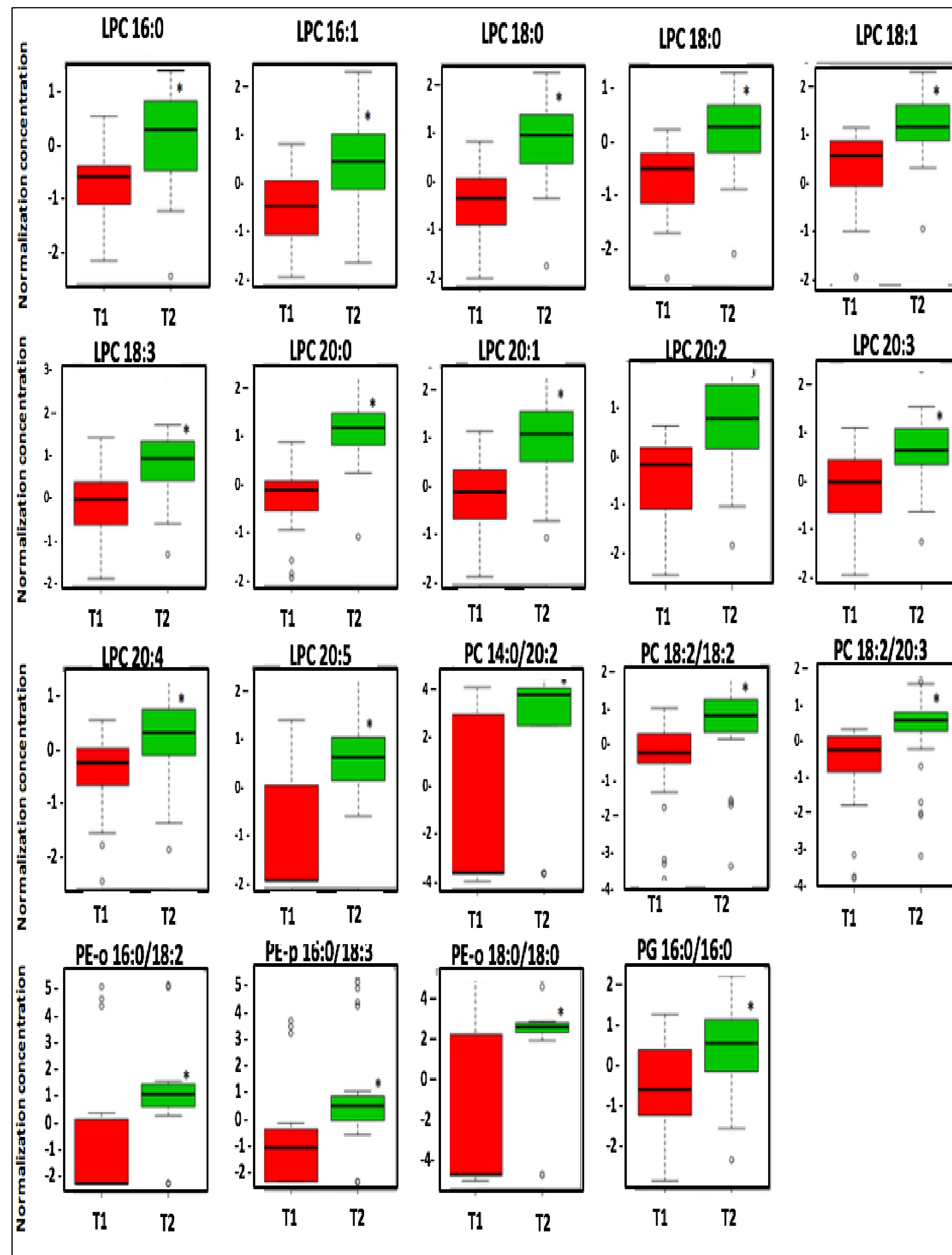

Figure 5: Specific lipids ch Conclusions and Future Directions Our study for the first time, identify the lipid differences pre-
ransplant and post-transplant. Additionally, we identify a pre transplant lipid signature that distinguish kidney transplant patients with favorable transplant outcomes (SC) and a major form of nonunlike SC patients that demonstrate a dynamic longitudinal lipid change, AMR patients maintain a relatively unchanging lipid profile over time with respect to the measured lipids. Finally, we demonstrate for the first time the potential for risk stratification of the potential for onset of AMR. Following validation in a larger cohort, these findings have the potential to alter the current paradigm of post-transplant monitoring and treatment of these patients via an evidence based risk stratification strategy and thereby vastly improving the success of kid

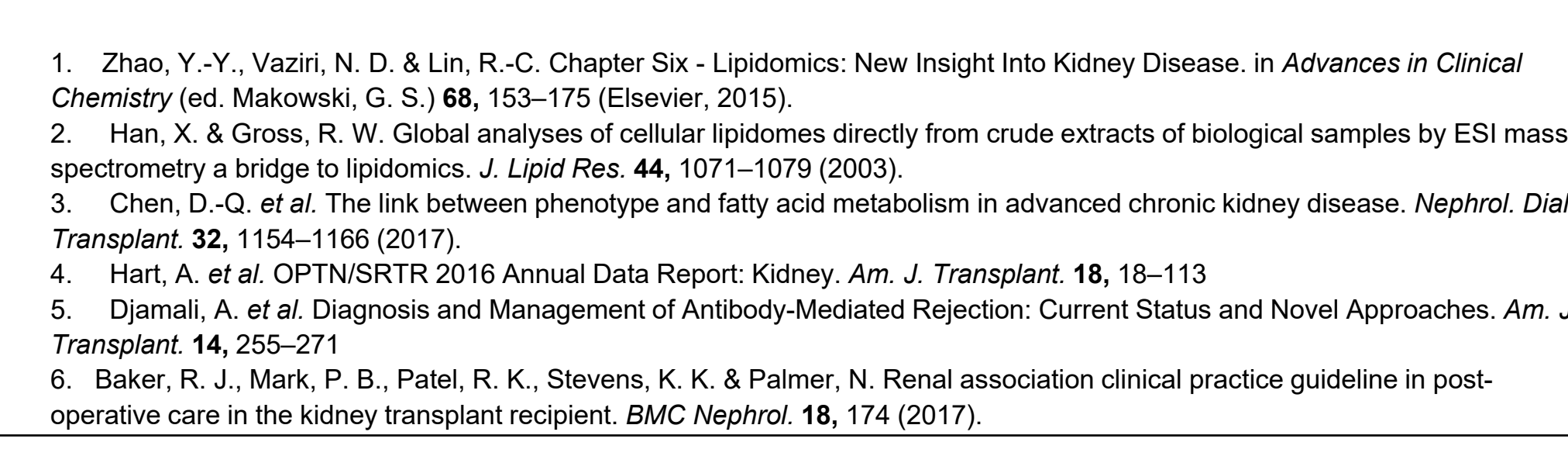

